\title{
Towards SKA studies of the fast radio transient Universe
}

\author{
Peter J Hall' ${ }^{1}$, Timothy Colegate, J. - P. Macquart, Nathan Clarke, Steven Tingay \\ and Randall Wayth \\ International Centre for Radio Astronomy Research (ICRAR) /Curtin University \\ E-mail: peter.halleicrar.org
}

Cathryn Trott

ARC Centre of Excellence for All-Sky Astrophysics (CAASTRO) /Curtin University

E-mail: cathyrn.trottecurtin.edu.au

\begin{abstract}
The SKA is likely to produce transformational insights into the fast transient Universe. So little is currently known about this observational window that much of the SKA time domain science program has been lumped into the "exploration of the unknown" theme. Unsurprisingly, no clarity has hitherto emerged on the associated operational or architectural requirements. However, with the recent advent of highly-capable Pathfinders and new programs targeted at exploring key system design questions, insights into these and other critical issues are emerging. Newly-optimized signal combination strategies for large- $\mathrm{N}$ arrays, cost effective hardware processing methods, and improved signal detection and localization algorithms are all being refined and implemented on test platforms. Somewhat unexpectedly, the power of SKA-low as a fast transients instrument has recently become evident. This paper reviews a new detectionrate metric developed at ICRAR/Curtin, summarizes the present state of transients surveys, predicts SKA performance, and outlines important messages for the SKA project - particularly the SKA1 system design now underway.
\end{abstract}

Resolving the Sky - Radio Interferometry: Past, Present and Future

Manchester, $U K$

April 17-20, 2012

\footnotetext{
1 Speaker
} 


\section{Introduction}

The systematic study of fast transients $(<5 \mathrm{~s}$ duration) is a relatively new sub-field of radio astronomy. Although isolated candidate detections have appeared over the years, the detection and/or verification arrangements have not hitherto allowed an unambiguous detection of a cosmic event. Many of the candidate detections, such as that of Lorimer et al., [1] have emerged from pulsar surveys, these surveys being the best probe to date of promising parameter space. Excitement levels are currently high: fast transients offer insights into (for example) the physics of extreme states of matter, high brightness emission mechanisms, and the physics of strong gravitational fields. Detection of extragalactic events also promises a new view of the huge reservoir of baryons in the ionized intergalactic medium.

Complementing the current scientific interest in transients is the availability of new trailblazer and SKA Pathfinder experiments which, as we show later, give cause for optimism in the search for astronomically interesting events. While fast transients still fall under the "exploration of the unknown" heading in SKA science discussions, the present level of activity by several groups - including those associated with the ATA [2] and LOFAR [3] - will grow rapidly the maturity of the field. Such growth is essential in uncovering the richest lodes in parameter space, and in informing technical and investment plans for the SKA. In this short paper we confine commentary largely to work undertaken by ICRAR and collaborators, the main aim of which is to shed light on key science and engineering aspects of SKA transients observing.

\section{Parameter space}

While many have speculated on the types of events which may produce fast transients, our lack of knowledge about population characteristics (e.g. luminosity distributions) and locations makes it incumbent to explore as much observing parameter space as feasible. Search parameters include frequency, field-of-view (FoV), limiting sensitivity, dispersion measure, and polarization. The major challenge in detecting and verifying transients is the one-off, or nearly one-off, nature of putative phenomena. Rather than referring to traditional radio astronomy metrics, figures of merit relating to what the military and others call "probability of intercept (PoI)" are more meaningful. Factors influencing PoI include receptor sensitivity, array or subarray sensitivity (if applicable), FoV, observing duration (maximimized with commensal programs) and detector characteristics; the latter include time resolution and the goodness of matched filter approximations in the frequency and time domains.

Many of the PoI factors are strong functions of technology, exemplified by ever more capable telescopes or instruments. We expect the nexus between discovery and new technology to hold and are optimistic about prospects for transients astronomy in the SKA Pathfinder era.

The uncertain population characteristics of transient sources and the availability of many signal combination modes in large- $\mathrm{N}$ arrays (many antennas) have hitherto made it impractical to formulate other than "blind" observing plans. Recently we have begun to explore the connection between putative cosmic populations and various instrumental signal combination 
and processing modes. Our chosen metric, closely related to PoI, is event rate: a good telescope produces a large number of detected events. This analysis environment allows us to (a) maximize detections from given putative populations and, conversely, (b) understand the implicit filtering of observed populations resulting from e.g. opportunistic signal combination strategies.

\section{Event rates}

The formalism involving event rate as a figure of merit has been set out by Macquart [4]. It relates the expected detection rate by a telescope with particular key characteristics (notably limiting sensitivity and FoV) to the properties of a cosmic population of fast transient sources. A key equation from the analysis is:

$$
\mathcal{R}_{v}=\frac{1}{3} \rho \Omega_{\operatorname{proc}_{v}}\left(\frac{W_{\mathrm{i}}}{W_{v}}\right)^{\frac{3}{4}}\left(\frac{\mathcal{L}_{\mathrm{i}, v}}{4 \pi S_{\min _{v}}}\right)^{\frac{3}{2}}
$$

where $R_{v}$ is the observed event rate, $\rho$ is the intrinsic event rate density (i.e. the number of events per cosmic volume per second), $\Omega_{\text {procv }}$ is the instantaneous processed FoV, $\mathcal{L}$ is the intrinsic luminosity of the object, and $\mathrm{W}_{\mathrm{i}} / \mathrm{W}_{\mathrm{v}}$ is the ratio of the intrinsic pulse width to its observed duration. The parameter $S_{\text {min }} \propto T_{\text {sys }} / A_{\text {eff }}$ is the lowest detectable flux density on the timescale of the transient's emission, and embodies a multitude of telescope and sky-based sensitivity considerations.

The event rate formalism allows one to quantitatively assess the impact of trade-offs between FoV and sensitivity. The method by which signals from antennas and stations are combined influences the efficiency of the transients search by impacting the balance between these two quantities. A variety of situations can be modelled and Figure 1 (below) shows the Galactic event rate at $1 \mathrm{GHz}$ given a number of scientifically interesting assumptions. One can use this formalism to decide whether, for a fixed amount of processing capability, it is better to search a finite number of high-sensitivity, small FoV, tied-array beams, or to combine station beams incoherently in order to maximize FoV at the expense of sensitivity. The formalism can also be employed to address the optimal telescope configuration; the intra-station and array station layouts impact heavily on the trade-off between FoV and sensitivity.

In the above equation, the dependence $\mathrm{R}_{v} \propto \mathrm{S}_{\min }{ }^{-3 / 2}$ stems from the assumption that the events are distributed homogeneously throughout the detection volume and that their flux density falls as the inverse square of distance. This dependence on $S_{\min }$ is softened if the events are confined within a finite volume (e.g. the Galaxy) or are subject to temporal smearing caused by interstellar (or possibly intergalactic) scattering. When scattering is important the increase of $R_{v}$ is moderated because, as $S_{\min }$ decreases, we probe to larger distance and thus encounter more scattering material, so the signal-to-noise ratio of a transient of any given luminosity will decay faster than the inverse-square of distance. This is an important consideration in the design of transients surveys at the frequencies spanned by the aperture array component of the SKA, where interstellar scattering will influence the observable properties of fast transients 
significantly. At a practical level it means that simply increasing sensitivity does not necessarily, even at $\sim 1.4 \mathrm{GHz}$, increase the event rate; for some Galactic surveys in particular, coherent modes never offer an advantage and the larger FoV of incoherent surveys is always preferable.

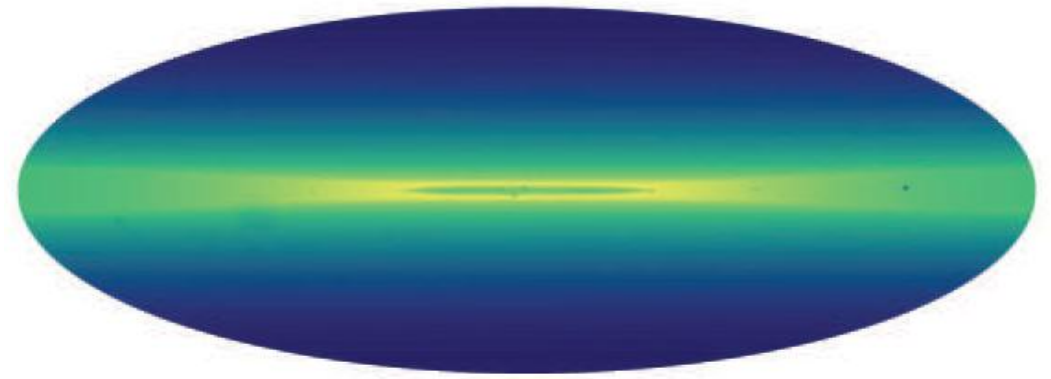

Figure 1. The normalized event rate in the Galaxy as a function of Galactic position for a hypothetical population of transients [4]. The plot is colour coded according to the $\log _{10}$ of the event rate. Note that the rate drops near the Galactic centre due to the effects of interstellar scattering. For the population modelled the minimum detectable flux density is $\mathrm{S}_{\min }=10 \mathrm{mJy}$, and the minimum and maximum luminosities are $0.1 \mathrm{Jy} \mathrm{kpc}^{2}$ and $100 \mathrm{Jy} \mathrm{kpc}^{2}$ respectively, with a power luminosity distribution with index -1.5 .

\section{State of the survey art}

Colegate and Clarke [5] have produced a summary (Table 1) of recent or forthcoming fast transients surveys, summarizing the expected detection rate on an "event rate per beam" basis, a measure allowing the processing efficiency and cost of transient survey strategies to be compared and optimized. In some cases both coherent and incoherent modes are possible and a representative performance for each case is noted.

\begin{tabular}{|c|c|c|c|c|c|c|}
\hline Experiment $^{\mathrm{a}}$ & $\begin{array}{l}\text { Telescope } \\
\text { and status }\end{array}$ & $\begin{array}{r}v_{\text {centre }} \\
(\mathrm{MHz})\end{array}$ & $\begin{array}{c}\Delta v \\
(\mathrm{MHz})\end{array}$ & $\begin{array}{l}\text { Max. baseline } \\
(\mathrm{km})^{\mathrm{b}}\end{array}$ & $\begin{array}{l}\mathcal{R}_{\text {beam }}{ }^{-1} \\
\text { (normalised) }^{\mathrm{c}}\end{array}$ & $\begin{array}{l}\text { Max. beams } \\
\text { available }\end{array}$ \\
\hline Archival searches $^{\mathrm{d}}$ & Parkes & N/A & - & - & - & - \\
\hline High Time Resolution Universe Pulsar Survey ${ }^{\mathrm{f}}$ & $\begin{array}{l}\text { Parkes } \\
\text { (operational) }\end{array}$ & 1352 & 340 & N/A & $10^{-2}$ & 13 \\
\hline V-FASTR ${ }^{\text {h }}$ & $\begin{array}{l}\text { VLBA } \\
\text { (operational) }\end{array}$ & 1400 & 64 & 6000 & $10^{-2}$ (inc.) & 1 \\
\hline LOFAR Transients Key Science Project ${ }^{\mathrm{i}}$ & $\begin{array}{l}\text { LOFAR } \\
\text { (in progress) }\end{array}$ & 120 & 32 & $<100$ & $\begin{array}{l}10^{-1} \text { (inc.) } \\
10^{-4} \text { (coh.) }\end{array}$ & $\begin{array}{l}1^{\dagger} \\
\text { thousands }{ }^{\dagger}\end{array}$ \\
\hline $\begin{array}{l}\text { Commensal Real-Time ASKAP } \\
\text { Fast-Transients (CRAFT) Survey }^{j}\end{array}$ & $\begin{array}{l}\text { ASKAP } \\
\text { (planned) }\end{array}$ & 1400 & 300 & 6 & $\begin{array}{l}10^{-2} \text { (inc.) } \\
10^{-6} \text { (coh.) }\end{array}$ & $\begin{array}{l}36 \\
\mathrm{~N} / \mathrm{A}\end{array}$ \\
\hline SKA1 low band dishes & & 725 & 550 & 200 & $\begin{array}{l}1 \text { (inc.) } \\
10^{-2} \text { (coh.) }\end{array}$ & $\begin{array}{l}1 \\
\text { thousands } s^{\dagger}\end{array}$ \\
\hline
\end{tabular}

Table 1. Recent, current and forthcoming fast transients surveys. Refer to [5] for details and footnotes. 
Bearing in mind the concept of probability of intercept, we have attempted to codify the parameter space covered by past, present and future surveys. Figure 2 displays the coverage in terms of minimum detectable flux density, observing time and FoV for $\sim 1.4 \mathrm{GHz}$ surveys. One-hour observations with given telescopes are indicated, together with the limits reached by longer observations (commonly $5000 \mathrm{hr}$, or $\sim 1$ year). Superposed on the plot are diagonal lines representing (a) expected FoV-time product and $S_{\min }$ required to detect a single event from populations of Crab-like giant pulse emitters at the Virgo Cluster and M33, and (b) the limits on the intrinsic event rate density (in events per hour per cubic mega-parsec) which one might infer from detecting a single Crab-like event from a homogenous extragalactic population. The shaded region represents the parameter space notionally excluded by the PALFA survey, the survey with the lowest limiting sensitivity to date. Here, our extragalactic giant pulses are assumed to occur with a frequency of $0.04 \mathrm{~s}^{-1}$, for pulses with minimum energy of $5 \mathrm{kJy} \mu \mathrm{s}$ (at $2 \mathrm{kpc}$ ), a power-law energy distribution (index $=-2.6$ ) and no known maximum energy. Figure 3 shows the geometry and main assumptions in each case.

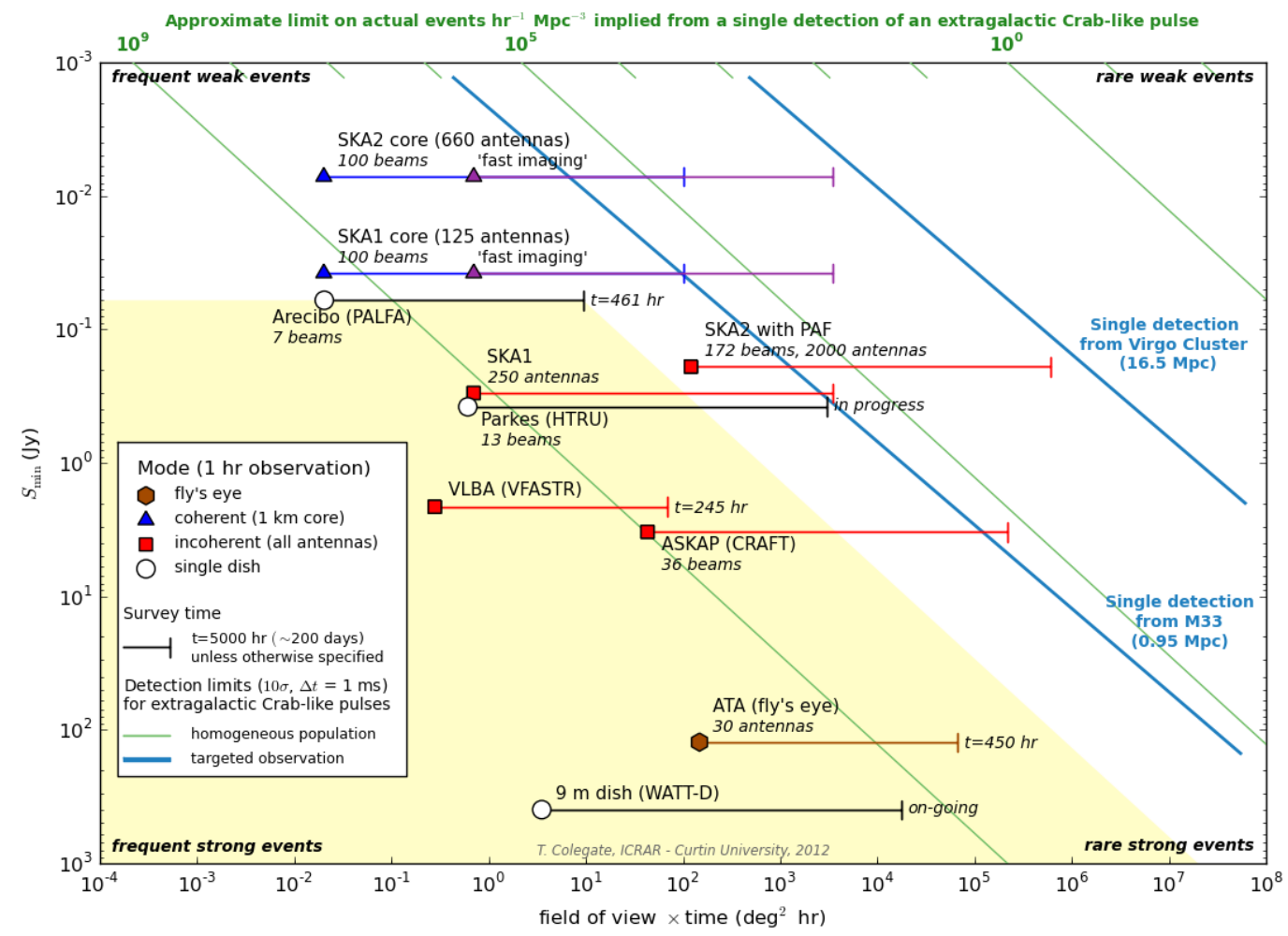

Figure 2. Parameter space at $\sim 1.4 \mathrm{GHz}$ for selected telescopes and surveys. Refer to text for details.

Figure 2 encapsulates a great deal of information but a very important point is that SKA1 and even Pathfinder experiments are plausibly capable of detecting astronomically interesting events at extragalactic distances, although the power of SKA2 is required for sources beyond $10 \mathrm{Mpc}$. In PoI terms the continuing Parkes HTRU survey is highly competitive but a truth not captured by the plot is that event verification, and false detection exclusion, is much better 
achieved by arrays operating either in conventional interferometry or spatial diversity (timing) modes. Our continuing work seeks to quantify the array advantage in statistical terms.

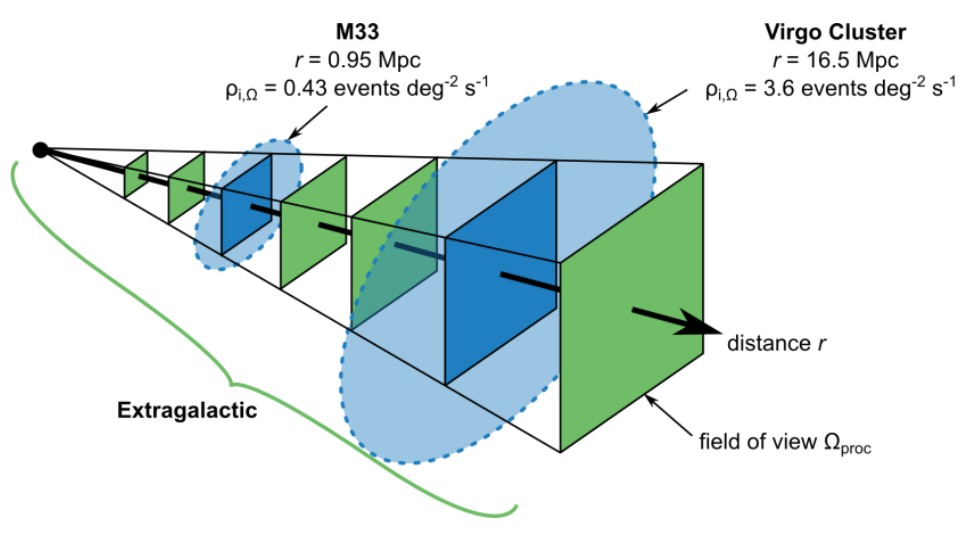

Figure 3. Geometry of detecting Crab-like giant pulses, for populations located at a fixed distance (blue), or a homogenous extragalactic population (green). The intrinsic event rate per solid angle $\rho_{\mathrm{i}, \Omega}$ is extrapolated from Crab giant pulse statistics and the number of target galaxies.

Figure 2 hints at other profitable areas of investigation. For example, one clearly requires a minimum sensitivity to begin accessing emitter populations at given distances. Conversely, the lower right of the plot implies a role for near-continuous observation using relatively insensitive telescopes. While on-going observations are necessary to set observational limits, one eventually reaches a region requiring events of extraordinarily high brightness temperature in order to make detections possible. Invoking known physics excludes areas of the plot and we see scope for examining these issues theoretically, in parallel with new-generation observations with SKA Pathfinders.

\section{Trailblazers, Pathfinders and innovation}

Nothing better illustrates the nascent phase of the fast transients sub-field than the current need for pre-Pathfinder experiments to refine system and instrumentation design. A number of these "trailblazer" experiments are underway internationally at instruments such as LOFAR, MWA and the VLBA. We have two active trailblazers, both being developed with international colleagues but each using different technologies and observing platforms. Their common aims are:

- as science activities, to examine potentially interesting regions of fast transients parameter space;

- as engineering projects, to explore scalable architectures and algorithms for Pathfinders and, eventually, the SKA; and

- as information processing experiments, to assess the aplicability of new, pipeline based algorithms and techniques for SKA-era instruments.

Our most developed trailblazer, V-FASTR, is a VLBA software instrument based on incoherent signal combination, with detection verification and event localization via triggered correlation of buffered, coherent signals. V-FASTR is a collaboration between ICRAR, JPL, 
ASTRON and NRAO and is described in detail by Wayth et al. [6] and Thompson et al. [7]. With 1300 hours of commensal observing to date, the experiment has already produced scientifically interesting event rate limits [8]. A particularly interesting V-FASTR feature is the application of machine learning techniques to flag statistical anomalies in very large data sets. Used at first in parallel with more conventional approaches, machine learning and associated techniques are producing results which promise good receiver operating characteristics (high true/false detection rates) in a pipeline analysis environment.

Our second trailblazer, TARDIS, is an FPGA-based transients processor developed for ASKAP, partly under the auspices of the CRAFT survey science project [9]. TARDIS uses incoherent signal combination from all 36 dishes prior to event detection, thereby retaining the full $30 \mathrm{deg}^{2} \mathrm{FoV}$ of the ASKAP phased array feeds. An important part of the system is a fast FPGA de-disperser capable of implementing efficient new algorithms of the type described in [10]. Hundreds of trial dispersion measures (up to DM 10 000) will be available during realtime processing of the $300 \mathrm{MHz}$ ASKAP bandwidth; maximum time resolution is < $1 \mathrm{~ms}$. TARDIS has been developed jointly by JPL and ICRAR and first deployment, most likely on a DSN antenna, is expected in late 2012.

\section{SKA1}

Fast transients are not currently headline science in SKA1 but Figure 2 shows that the telescope has considerable discovery promise in this domain, especially when its event localization and commensal observing potential are accounted for. While trailblazers and Pathfinders will clarify the appropriate level of SKA1 fast transients investment, it is encouraging that an "entry level" transients mode can be incorporated for minimal cost. Figure 4 shows a dish-based system, employing a variant of LOFAR-style incoherent signal combination followed by coherent localization. Using projections based on Uniboard correlators [11] we estimate that the total incremental cost of the SKA1 transients system (including processor, memory buffers and storage) would be of order $€ 1 \mathrm{M}$. The transients processor is a hybrid CPU/FPGA type, offering more than 500 trial dispersion measures extending beyond $\mathrm{DM}=5000$. The system requires an SKA1 data spigot and in-line buffer, both of which are likely to be required for other reasons. Indeed, the "spigot + instrument" philosophy is central to sensible SKA development, providing a large measure of obsolescence protection. 


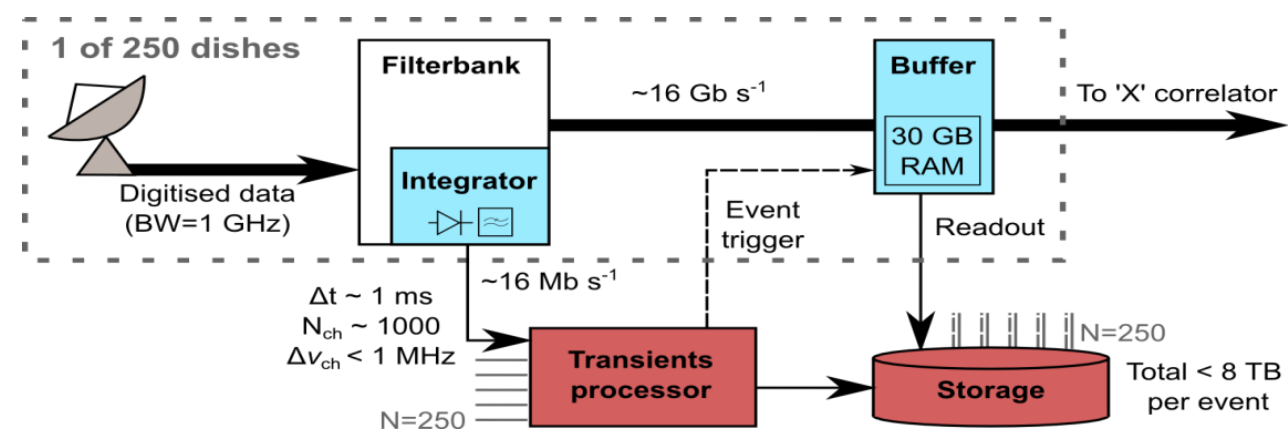

Figure 4. Representative transients procssing system for the SKA1 dish array. The transients processor accepts integrated spectrometer data and, on detection of an event, triggers the recording of coherent data samples for later correlation and localization.

Leaving the dish example, the event rate per beam metric mentioned in Section 4 indicates that SKA1-low will also be a highly efficient transients telescope. Figure 5 shows this metric plotted as a function of frequency and line-of-sight for two different source spectral indices. For other than observations close to the Galactic plane, SKA1-low will be a very effective transients telescope and one can envisage a wealth of signal combination modes (including coherent modes) not useful with the extremely sparse dish array.
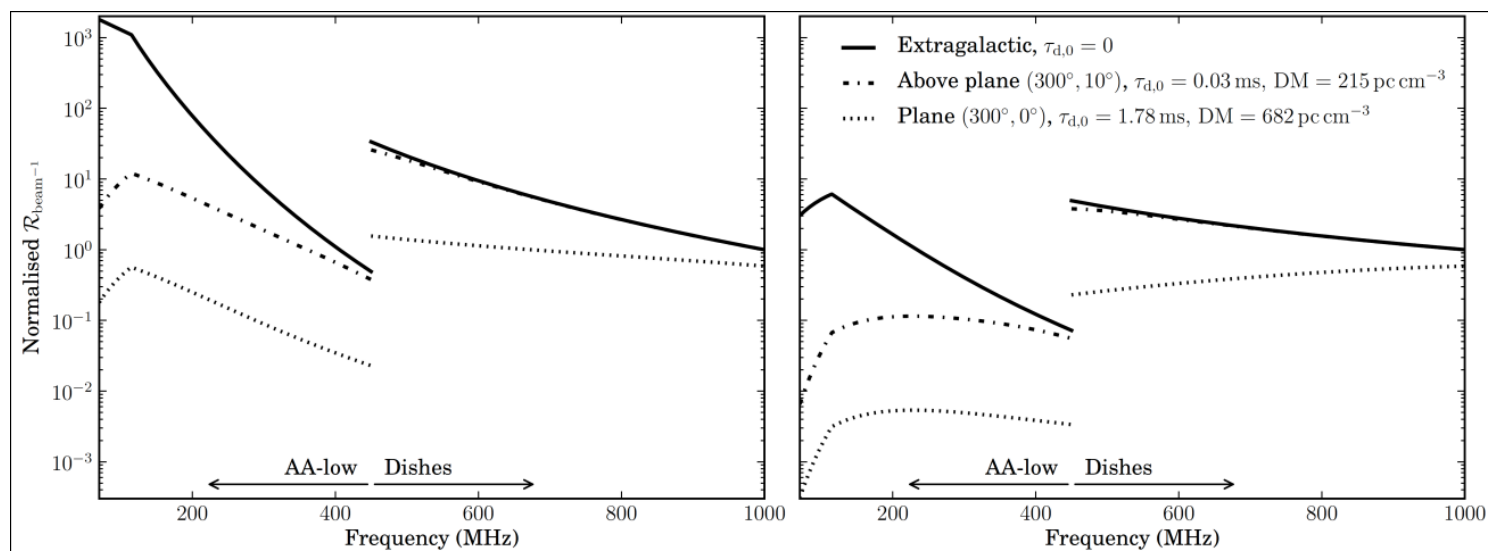

Figure 5. Normalised event rate per beam for SKA1 incoherent combination mode, for source spectral index of -1.6 (left) and 0 (right). For a coherent combination of the element in the SKA1 core, the event rate is less by a factor of $\sim 5$ (SKA1-low) and $\sim 157$ (dishes). Refer to [5] for details.

\section{Conclusion}

After tanatalizing hints, confirmed detection of a fast cosmic transient would be welcome and significant. Existing telescopes have begun to probe scientifically interesting regions of parameter space and, with the era of SKA Pathfinders upon us, the next few years should confirm the value of investing in a transients capability for SKA1 and SKA2. Apart from finding the way forward scientifically, the Pathfinders provide invaluable platforms for innovations in signal processing, algorithms, architectures and high-volume analysis pipelines. 


\section{References}

[1] D. Lorimer, M. Bailes, M. McLaughlin, D. Narkevic, F. Crawford, A bright millisecond radio burst of extragalactic origin, Science 318, 777 (2007)

[2] A. Siemion, G. Bower, G. Foster, P. McMahon, M.Wagner, D. Werthimer, D. Backer, J. Cordes, and J. van Leeuwen, The Allen Telescope Array fly's eye survey for fast radio transients, Ap. J. 744,109 (2012)

[3] B. Stappers et al., Observing pulsars and fast transients with LOFAR, Astron. and Astrophys., 530 (2011).

[4] J. - P. Macquart, Detection rates for fast transients with next generation radio telescopes, Ap. J. 734, 1 (2011)

[5] T. M. Colegate and N. Clarke, Searching for Fast Radio Transients with SKA Phase 1, PASA 28, (2011)

[6] R. Wayth, W, Brisken, A. Deller, W. Majid, D. Thompson, S. Tingay and K. Wagstaff, $V$ FASTR: The VLBA fast radio transients experiment, Ap. J. 735, 97 (2011)

[7] D. Thompson, K. Wagstaff, W. Brisken, A. Deller, W. Majid, S. Tingay and R. Wayth, Detection of fast radio transients with multiple stations: a case study using the Very Long Baseline Array, Ap. J. 735, 98 (2011)

[8] R. Wayth, S. Tingay, A. Deller, W. Brisken, D. Thompson, K. Wagstaff and W. Majid, Limits on the event rates of fast radio transients from the V-FASTR experiment, Ap. J. Letters, submitted (2012).

[9] J. - P. Macquart, P. Hall and N. Clarke, The art and design of the CRAFT survey, PoS 039 (2010)

[10] N. Clarke, J. - P. Macquart and C. Trott, Performance of a novel fast transients detection system, Ap. J., submitted (2012)

[11] A. Szomoru, P. Boven, J. Hargreaves, S. Pirruccio, S. Pogrebenko, A. Gunst and G. Schoonderbeek, A Uniboard-based phase 1 SKA correlator and beamformer concept description, SKA Signal Processing CoDR document WP2-040.070.010-TD-001 Rev 1, SPDO (2011) 